\title{
Compliance and confirmation bias on the emergence of cultural conventions
}

\author{
José Segovia-Martín ${ }^{1,2, *}$ \\ ${ }^{1}$ Complex Systems Institute (ISC-PIF), CNRS, Paris \\ ${ }^{2}$ M6 Polytechnic University, Benguerir \\ *jose.segovia@um6p.ma
}

\begin{abstract}
In the present study we develop a co-evolutionary model of cardinal preferences and institutions to explore how the dynamics of cultural diversity in populations with different levels of compliance and confirmation bias evolve. This is the first attempt to formalise these two types of bias in a single learning algorithm for agents learning in iterative chains without access to complete information. Results show that, in some regions of the parameter space, institutional influence facilitates the emergence of shared cultural conventions when compliance biases increase. In general, a compliance bias pushes diversity up when institutions are diverse, and pushes diversity down when institutions convey value systems with strong dominance of one or few cultural variants. Interestingly, in some scenarios, a decrease in institutional influence and compliance bias allows the emergence of cultural conventions from the mutual reinforcement of local interactions and institutional values. We asses the robustness of these results by examining how sensitively they depend on different initial conditions of variant assignment, population sizes and alpha diversity indexes. Keywords: cultural evolution; co-evolution; institutions; value systems; cognitive biases; compliance bias; confirmation bias;
\end{abstract}

\section{Introduction}

One thing that makes humans special is their remarkable capacity to cooperate and build complex niches ${ }^{1-3}$. As a result of social learning, which involves learning through copying and sharing information with others, humanity has succeeded to construct institutional environments which interact with the individuals' cognition, affecting evolutionary dynamics and the distribution of culturally transmitted traits in a population ${ }^{4}$. Yet there are very few models that explore the co-evolutionary dynamics of institutional diversity and population cultural conventions at individual, institutional and historical levels by incorporating a comprehensive set of relevant learning biases. Therefore, it remains unclear how populations with a different characterization of biases and institutions with different degrees of influence and interests, all together, co-evolve social conventions and cultural diversity over time.

Although it has been shown that social conventions can emerge without the intervention of institutional mechanisms $^{5}$, prominent theories of collective behavior suggest that institutions reduce cultural diversity by facilitating the emergence of social conventions ${ }^{6-9}$. It is widely recognised that these variance-reducing institutions constitute niches, that is, modified environments which in turn are capable of altering sources of variation such as individual cognition and collective behaviours ${ }^{4,10,11}$. Notwithstanding, the nature of the institution itself (i.e. whether the institution conveys diverse values or hegemonic values) and the institutional capacity to transmit values to the population might interact in ways that potentially amplify or reverse the mentioned sources of variation and the formation of social conventions over time. In this study, we seek to answer these questions by using a co-evolutionary model.

Evidence gathered over recent decades overwhelmingly indicates that cognitive diversity varies substantially across human populations ${ }^{12-14}$. Understanding such source of variation implies understanding how transmission biases affect the spread of cultural variants during transmission processes ${ }^{15,16}$. We know that biased reasoning strategies can be adaptive and yield fitness advantages when compared to unbiased ones ${ }^{17}$. A number of these biases have been defined and modelled ${ }^{15,18}$. However, despite the importance of biased transmission in cultural evolution, there is a lack of formal co-evolutionary models integrating a diverse range of cognitive tendencies for the 
exploration of the complex dynamics of value systems and institutions.

Related to biased adoption and transmission of variants, we investigate three relevant oppositions between biases which play an important role in the maintenance of cultural diversity, namely: (i) A disproportionate tendency to copy the institutional value system (captured by an emergent institution) vs. a tendency not to conform to institutional values; (ii) Deeply-entrenched beliefs vs. weak beliefs; and (iii) A preference for content vs. a preference for variants that are more frequent in memory.

We operationalise these oppositions in terms of mathematical complements. The first opposition is characterised here as a bias to resist or conform to institutional values. The term compliance has been used in social influence studies to characterise the individuals' adherence to social norms, beliefs, acts and values in a broad sense; for a review see Cialdini and Goldstein, 2004 ${ }^{19}$. A related factor used in social science is authority bias, but in this study we will use compliance because it does not imply that the institution is necessarily authoritarian. Compliance bias is also related to positive frequency-based biases, such as conformist bias- 'follow the majority' ${ }^{20-23}$. In scenarios where our agents share cultural values to a high extent, compliance is very similar to conformity; however, when different sub-populations have different value systems, the analogy does not apply.

We also examine the tendency to adhere to one's personal beliefs, which play an important role in the dissemina-

tion of culture ${ }^{24,25}$. A value system can be thought of as a set of personal beliefs or hypotheses that affect behaviour. It can be operationalised as a set of values that agents assign to each cultural variant they encounter throughout their life and that they are capable to recall. When agents have deeply-entrenched beliefs they develop a tendency to recall information in a way that confirms their personal value system (henceforth confirmation bias), as opposed to agents that are more prone to consider new information to update their value systems. In this study, we operationalise confirmation bias as a tendency to process new information in a way that confirms one's prior beliefs ${ }^{26}$. Therefore, a non-confirmation bias corresponds to informational influence, that is a tendency to copy and reproduce information from others ${ }^{27}$.

In a previous study ${ }^{28}$, we examined the conditions under which convergence on shared opinions can be obtained in a social network where connected agents repeatedly update their normalised cardinal preferences (i.e. value systems) under the influence of a non-constant reflexive signal (i.e. institution) that aggregates populations' information using a proportional representation rule. Building on that model, here we develop a co-evolutionary model of institutions, value systems (as cardinal preferences) and choice that integrates compliance and confirmation bias into the agents' learning algorithm. We aim to establish the effect of these variables on the evolution of cultural diversity and the emergence of shared cultural conventions.

\section{Methods}

\section{The model}

We consider a simplified micro-society of agents, each of whom is characterized by a number of state variables as described in Table 1. The micro-society initially contains $N$ agents, who pair-up and interact for a number of rounds $(R)$. Each interaction consists of an exchange of cultural variants selected from an initial pool of variants $(V)$ (Figure 1).

Agent pairings are scheduled using a method that takes the sequence of agents of the population after each round, shuffles the order of agents and then aggregates the agents into pairs, so that the order of scheduling was randomized at each time step. Each simulation begins with $N$ agents, each initialised with a unique cultural variant and a value system. Agents' production of variants and their value systems evolve according to the model dynamics described in section . Population size was kept constant. Model runs proceeded in discrete time steps, called "rounds". For illustration purposes, a flowchart depicting relevant activity during one round is diagrammed in Figure 1. 


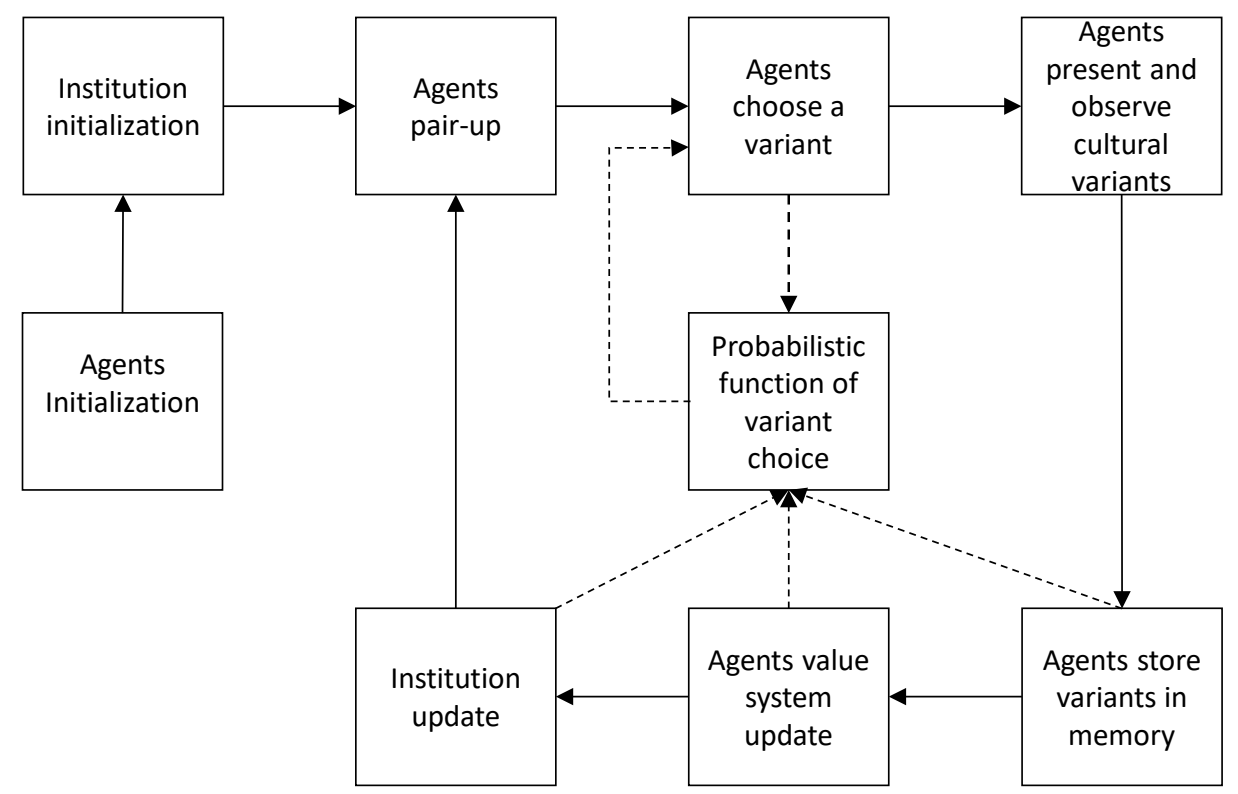

Figure 1. Flowchart depicting relevant activity during one round. Arrow direction represents the time-flow of events. Plain lines represent transition from one event to the following one. Dashed lines represent new data that is used to update agents prior information, affecting agents' variant choice over time.

\section{Standard model dynamics}

Let $X=\left\{x_{1}, x_{2}, \ldots, x_{V}\right\}$ be a vector of $V$ cultural variants (each variant represents a different kind of property, taste or behavior of an agent regarding a particular trait (e.g., language, norm, religion, music choice, etc.) and takes its value from the combination of a range of parameters (see Equation 3)), and let $A=\left\{a_{i}, a_{j}, \ldots, a_{N}\right\}$ be the set of agents in a population. In the initial state each agent $n \in A$ is randomly assigned a cultural variant $v \in X$ selected from $X$ with replacement, so that the model is not initialised with maximum diversity. Then, $x_{n v 0}$ is a random variant $v$ assigned to an agent $n$ at round $0(r=0)$. For example, at round $0, x_{i v 0}$ is a random variant initially assigned to agent $i, x_{j v 0}$ is a random variant initially assigned to agent $j$, and so on.

At the beginning of each round $r$, agents are paired randomly. Once agents are paired, they interact by presenting and observing one cultural variant (Figure 2). Agents' choice of variant $x_{n v r}$ to produce is sampled from its history (Figure 2, top) according to the probabilistic function defined in Equation 2. At this point, there is a small probability of innovation, in which case, the variant produced will be randomly sampled from one of the $V$ variants the population was initialised with. Both agents add both the produced and observed variants to their memories (Figure 2 , bottom). That is to say, at round $r$, when agent $i$ and agent $j$ interact, agent $i$ produces variant $x_{i v r}$ and agent $j$ variant $x_{j v r}$. Thus, at each round $r$, the total set of produced variants $X_{r}$ in the population is a vector of $N$ (one per agent) cultural variants $\left(x_{i v r}, x_{j v r}, \ldots, x_{N v r}\right)$, where $x_{i v r}$ is the variant produced by agent $i, x_{j v r}$ is the variant produced by agent $j$, and so on. 


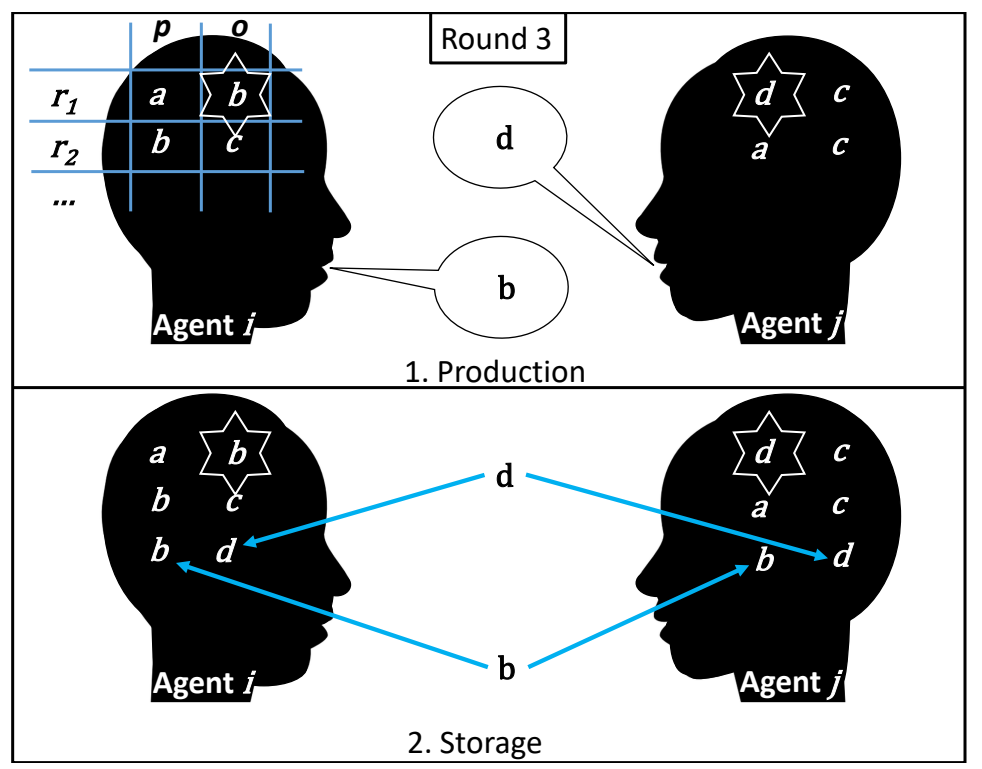

Figure 2. Illustration of the interaction between a pair of agents at one round of the simulation. At round 3, agents have already some variant tokens in memory, which they have stored in previous rounds. For each variant in memory, the letter represents the type (e.g. $a=x_{1}, b=x_{2}, \ldots$ ). Index $p$ represents variant tokens that were produced by self, while index $o$ are variants that were observed by the agent (variant produced by other agent). Index $r$ indicates the round at which the variant was stored. During the round depicted (round 3), first, one variant token in each agent's memory is selected for production (in the figure, the tokens surrounded by a star). Once both agents have produced a new token of their selected variant, they each proceed to store both in memory.

\section{State variables and probability distribution of variants}

The model takes several parameters as described below:

(a) Number of agents $(N)$ : We simulate micro populations of $N=10$ and $N=100$ agents. Each agent is initialised with a cultural variant randomly selected from a pool of $V$ distinct cultural variants with replacement, where $V=N$ at the beginning of each simulation. We also assess the robustness of our findings with respect to the initial variant assignment by examining random assignment without replacement.

(b) Number of rounds $(R)$ : Model runs proceeded in 100 rounds $r$. At each round, the pairing is randomized in such a way that each agent pairs up with another agent ( $N / 2$ pairs are formed).

(c) Value system $(S)$ : Each agent $n$ has a value system $S$, which is a vector of length $V$, which assigns a numerical value to each possible variant-choice. The state of the value system $S$ of agent $n$ at round $r$ can be defined as a vector that contains one value for each $V$ possible variant-choices, $\left(s_{n 1 r}, s_{n 2 r}, \ldots, s_{n V r}\right)$, where $s_{n 1 r}$ is the value assigned by agent $n$ to variant $x_{1}$ at round $r$, and $s_{n 2 r}$ is the value assigned by agent $n$ to variant $x_{2}$ at round $r$, and so on. Thus, $s_{n v r}$ is the value assigned by a random agent $n$ to a cultural variant $x_{v}$ at round $r . S$ assigns a number between 0 and 1 to each variant in $V$ (that is, there is a preference on $V$ represented by $s: V$ ). For example, if at round $0, S$ is $(1,0, \ldots, 0)$, then, at round 0 , agent $n$ assigns value 1 to variant $x_{1}$, value 0 to variant $x_{2}$, and so on. We examine two conditions:

(a) Pseudo-random (PR): In the initial state, each agent $n$ is assigned a value system $S$ so that the value of each variant is a random floating point number $N$ such that $0 \leq N \leq 1$.

(b) One takes all (OTA): Agents in the population are initialised with a hegemonic value system $S$ so that the value assigned to one preferred variant is 1 , and to the others 0 . 
(d) Institution $(G)$ : We assume that the micro-society is governed by a global institution $G$, which is a vector of length $V$. The state of the institution $G$ at round $r$ can be defined as a vector that contains one value for each $V$ possible variant-choices, $\left(g_{1 r}, g_{2 r}, \ldots, g_{V r}\right)$, where $g_{1 r}$ is the value assigned by the institution to variant $x_{1}$ at round $r$, and $g_{2 r}$ is the value assigned by the institution to variant $x_{2}$ at round $r$, and so on. $G$ assigns a numerical value between 0 and 1 to each possible variant-choice. For example, if at round $0, G$ is $(1,0.5, \ldots, 0)$, then, institution $G$ assigns value 1 to variant $x_{1}$, value 0.5 to variant $x_{2}$, and so on. At each round $r$, the institutional value $g_{v r}$ assigned to each possible variant-choice $x_{v r}$ is calculated as an arithmetic mean of the $N$ values $s_{i v r}, s_{j v r}, \ldots, s_{N v r}$, where $s_{i v r}$ corresponds to the value assigned by agent $i$ to variant $x_{v r}$, and $s_{j v r}$ corresponds to the value assigned by agent $j$ to variant $x_{v r}$, and so on. That is:

$$
g_{v r}=\frac{1}{N} \sum_{n v r=1}^{N} s_{n v r}=\frac{s_{i v r}+s_{j v r}+\ldots+s_{N v r}}{N}
$$

(e) Institutional influence $(\varepsilon)$ : It is a parameter that captures the capacity of the institution to effectively communicate its values to the agents. It takes values from 0 (null capacity) to 1 (full capacity).

(f) Compliance bias ( $\kappa)$ : It is a parameter that identifies the agent's bias to conform to institutional values. Its complement (a non-compliance bias $\kappa^{\prime}$ ) identifies a preference for the agents' own choice and value system, which can be (or not) different than institutional preferences. It takes values from 0 (null compliance) to 1 (full compliance).

(g) Confirmation bias $(\gamma)$ : It captures the tendency of agents to give stronger weight to their prior beliefs or hypotheses, which are encoded in the current value system of each agent. Thus, $\gamma$ identifies the tendency of an agent $n$ to assign value to a cultural variant $x_{v}$ according to its current value system $s_{n v r}$. Its complement $\gamma^{\prime}$ identifies a tendency of agent $n$ to assign value to a cultural variant $x_{v}$ according to its current choice, regardless of the current value system $s_{n v r}$. That is, when $\gamma$ is low, agents that are more prone to consider new information to update their value systems; $\gamma$ takes values from 0 to 1.

(h) Content bias $(\beta)$ encompasses two parameters $(b, d)$. Parameter $b$ is the agents' sensitivity to variant value $(s)$, and ranges from 0 -not sensitive at all-to 1 -fully sensitive-in steps of 0.1 . Parameter $d$ specifies whether the variant is in the agent's memory record, that is to say, whether the variant has been produced or seen at least once; $d$ is 1 if the variant is in memory, and 0 otherwise-in other words, one cannot prefer to re-produce a variant due to its quality until one has been exposed to that particular representation. Parameter $\beta$ is equal to $b \times d$. Thus, content bias $(\beta)$ assigns a value from 0 to 1 to each variant. Note that, at each round, as explained in the model dynamics, each agent stores two variants in its memory (the one that was produced by the agent itself and the one that was produced by its partner). Thus, content bias does not affect the storage process in our model (agents store in memory all the variants they encounter), it only affects the agents' production. In simple words, the higher the value of $\beta$, the higher the probability of producing a cultural variant due to its quality. When content bias is 0 , we have a neutral content model. Content bias values from 0 to 1 in steps of 0.1 are examined.

(i) Frequency bias $\left(\beta^{\prime}\right)$ : It is a parameter identifying an agent's preference for variants that are more frequent in its history. It corresponds to the complement of $\beta$. This parameter is limited by the agents' memory size $m$, that is, the maximum amount of history (in rounds) that can influence the variant choice. Each variant found in the history is a variant that was either produced or observed by the agent. A memory size of $m$ means that the model remembers the last $M$ variants $h \mid M, m$ from the history $h$. Given an agent $n$ and a variant $x_{v}$, then $f\left(x_{n v} \mid h_{n r \mid M, m}\right)$ is the relative frequency of variant $x_{v}$ in the memory of an agent $n$ by round $r$. Thus, $f\left(x_{n v} \mid h_{n r \mid M, 3}\right)$ corresponds to the relative frequency of variant $x_{v}$ in an agent memory for the last 3 rounds. Memory size between 2 and 4 rounds was associated with better model fit in ${ }^{29}$. 
(j) Innovation rate $(\mu)$ : Agents can generate novel variants. We fix the innovation probability at 0.02 by using a flat distribution $\phi(X)$ weighted by innovation rate, which means that $98 \%$ of variant choices would reflect the combined distribution (probability distribution yielded by all the parameters described above), while $2 \%$ would be a random choice among all $V$ initial variants in $X$. The probability level selected reflects the innovation rate found $\mathrm{in}^{30}$ and implemented $\mathrm{in}^{29,31}$.

\begin{tabular}{|c|c|c|c|c|}
\hline \multicolumn{5}{|c|}{ Model parameters } \\
\hline Entity & Parameter & Symbol & Number of levels & Value(s) \\
\hline \multirow{14}{*}{ Agent } & Content bias & $\beta$ & 11 & 0.0 to 1.0 in steps of 0.1 \\
\hline & Confirmation bias & $\gamma$ & 11 & 0.0 to 1.0 in steps of 0.1 \\
\hline & Compliance bias & $\kappa$ & 11 & 0.0 to 1.0 in steps of 0.1 \\
\hline & Memory & $m$ & 1 & 3 \\
\hline & Innovation & $\mu$ & 1 & 0.02 \\
\hline & Value system of an agent $n$ & $S=\left\{s_{n 1 r, \ldots, s_{n V r}}\right\}$ & & \\
\hline & Variant value (assigned by an agent) & $s$ & & Eq.3 \\
\hline & Agents' sensitivity to variant value $\mathrm{s}$ & $b$ & 2 & {$[0,1]$} \\
\hline & Variant in agent's memory record & $d$ & 2 & {$[0,1]$} \\
\hline & Agent' history & $h$ & & \\
\hline & Flat distribution of variants & $\phi(X)$ & & \\
\hline & Typical variant & $x_{v}$ & & \\
\hline & Typical agent & $n$ & & \\
\hline & Round & $r$ & & \\
\hline \multirow{9}{*}{ Global } & Initial set of agents & $A=\left\{a_{i}, \ldots, a_{N}\right\}$ & & \\
\hline & Initial number of agents per micro-society & $N$ & 3 & $10,50,100$ \\
\hline & Initial vector of cultural variants & $X=\left\{x_{1}, \ldots, x_{V}\right\}$ & & \\
\hline & Initial number of variants per population & $V$ & 3 & $10,50,100$ \\
\hline & Number of rounds & $R$ & 1 & 100 \\
\hline & Number of games per round & $N / 2$ & 3 & $5,25,50$ \\
\hline & Institution & $G=\left\{g_{1 r}, \ldots, g_{V r}\right\}$ & & \\
\hline & Institutional value (assigned by G) & $g$ & & Eq.1 \\
\hline & Institutional influence & $\varepsilon$ & 11 & 0.0 to 1.0 in steps of 0.1 \\
\hline
\end{tabular}

Table 1. Parameters, state variables and scales.

For each round in the simulation, for each agent, the model yielded a probability distribution of variants $(x)$ for a given history $(h)$ of previous rounds, according to the following equation. We use the apostrophe $(l)$ to denote the probabilistic complement: $a^{\prime}=1-a$.

$$
\operatorname{Pr}\left(x_{n v r} \mid h_{n r}\right)=\mu^{\prime} \beta^{\prime} f\left(x_{n v} \mid h_{n r \mid M, 3}\right)+\mu^{\prime} \beta s_{n v r}+\mu \phi(X)
$$

where $\operatorname{Pr}\left(x_{n v r} \mid h_{n r}\right)$ corresponds to the probability that an agent $n$ produces variant $v$ at round $r$ given the specific history of agent $n$ by round $r$.

We run each simulation with 14641 different parameter value combinations. For each parameter combination, we ran the simulation 200 times. For each model run we assume that all agents have the same connectivity dynamic, biases and memory sizes. The results below show the average and standard deviations of the number of runs of each parameter combination examined. All parameters and state variables can be found in Table 1.

\section{The co-evolution of variant-choice, value system and institutions}

The model is initialised with each agent having a cultural variant and a value system. At each round, the value assigned by each agent $n$ to variant-choice $x_{v}$ is updated according to the following equation, where, as already noted, the apostrophe ( $/$ ) denotes the probabilistic complement: $a^{\prime}=1-a$ : 


$$
s_{n v r+1}=g_{v r} \varepsilon \kappa+\left(x_{v r} \gamma^{\prime}+s_{n v r} \gamma\right) \kappa^{\prime}
$$

where $s_{n v r+1}$ is the value assigned by an agent $n$ to a variant $x_{v}$ in the next round $r+1, g_{v r}$ is the institutional value assigned by an institution to a variant $x_{v r}, \varepsilon$ stands for institutional influence, $\kappa$ for compliance bias, $\gamma$ is a parameter that captures the relative weight of the current value system $s_{n v r}$ in its future value system $s_{n v r+1}$, in a way that affirms the agent's prior value system or hypothesis (or confirmation bias), and $x_{v r}$ represents the target variant, which takes value 1 if it has been produced in the current round, and 0 otherwise. Since one agent might eventually produce cultural variants which do not match its current value system but which inform its future value system, $\gamma^{\prime}$ captures the relative weight of current choices in the future variant value $s_{n v r+1}$ at round $r+1$, regardless of the current variant value $s_{n v r}$. For illustration purposes, Figure 3 shows some characteristic parameter combinations that satisfy three different solutions for Equation 3.

As explained above, by implementing this algorithm our model is able to update agent's value systems from round to round. In turn, the form of the institutional value system is also updated at the beginning of each round. The co-evolution of institutional value systems and individual value systems model the value $s$ assigned by each agent to each possible variant over time. 


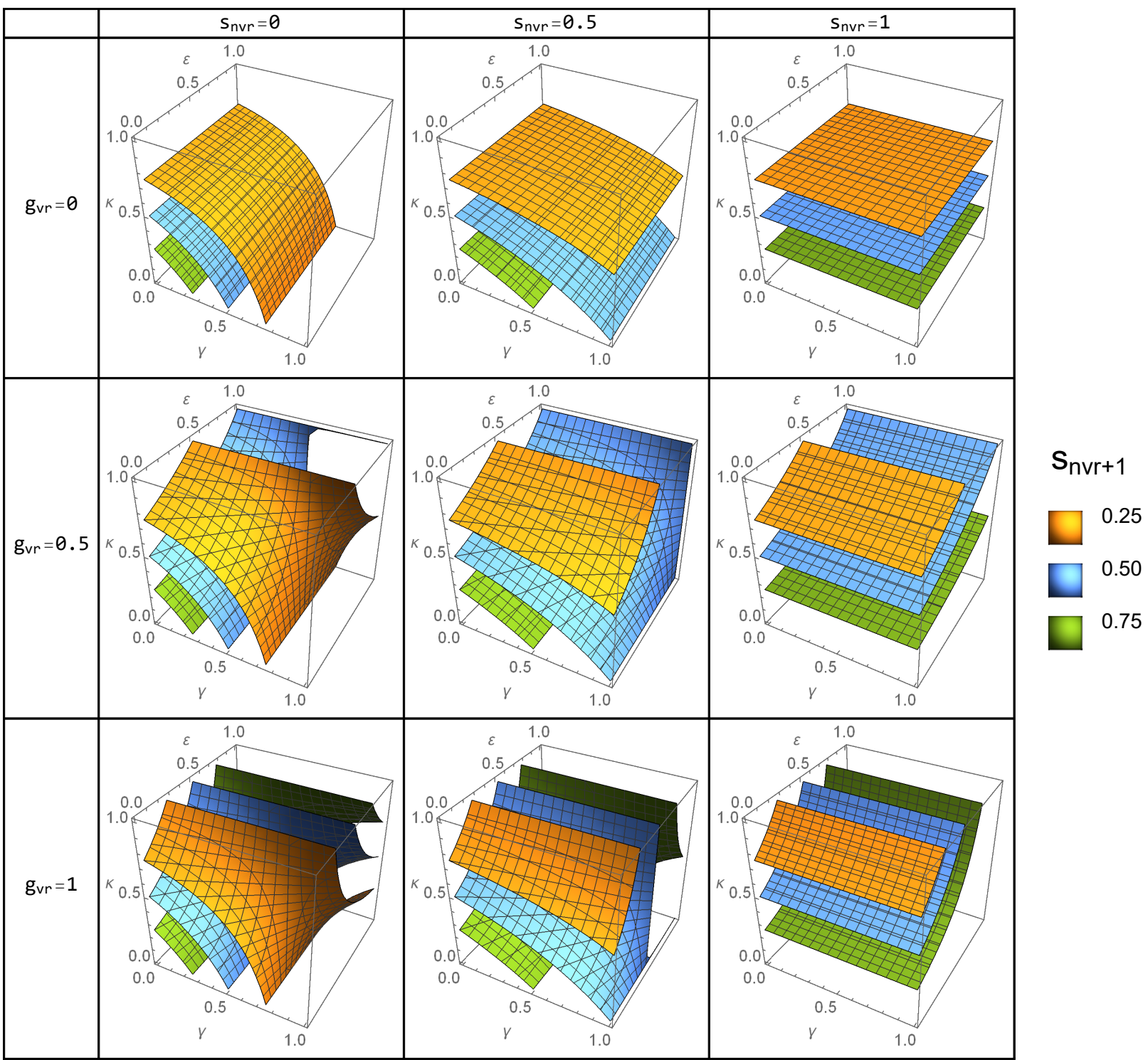

Figure 3. Illustration of some parameter combinations in the 3D space. Given that an agent $n$ produces variant $x_{v}$ at round $r$, plots show all possible combinations of values of institutional influence $(\varepsilon)$, compliance bias $(\kappa)$ and confirmation bias $(\gamma)$ that satisfy $s_{n v r+1}=0.25,0.5$ and 0.75 , according to Equation 3, for three different levels of $g_{v r}$ and $s_{n v r}$. For example, if at round $r$ a variant $x_{v}$ is assigned value $g_{v r}=0$ by an institution and value $s_{n v r}=1$ by an agent $n$ with compliance bias $\kappa=0.5$, that means that the value assigned by the agent to variant $x_{v}$ at round $r+1$ will be $s_{n v r+1}=0.50$. Lower compliance bias ( $\left.\kappa\right)$ tends to contribute more with variant value when institutional values $(g)$ are weak (see top right). When institutional values are stronger and agent' values $(s)$ are weak, confirmation bias and institutional influence tend to invert the pattern of contribution to variant value (see bottom left). 


\section{Quantifying cultural diversity}

In the present chapter, we are interested in the diversity of agents' produced variants and in the diversity of institutional values at each time step. As in previous chapters, we use Shannon's entropy to quantify diversity. In order to facilitate the comparison with other metrics and to eliminate the effect of different population sizes and time series, we normalise entropy by $\log _{2} n$ to obtain $H_{n}(V) \in[0,1]$ :

$$
H_{n}(V)=-\sum_{v_{i} \varepsilon V} \frac{p\left(v_{i}\right) \log _{2} p\left(v_{i}\right)}{\log _{2} n}
$$

where $V$ corresponds to the set of variants, and $p\left(v_{i}\right)$ is the probability of $i^{\text {th }}$ variant in that set, and $n$ is the number of variants. High entropy corresponds to high diversity and also to low convergence on shared conventions.

We also use a Simpson's index. Instead of taken the Simpson's reciprocal index, we use the Simpson's diversity index in order to facilitate the comparison with normalise entropy when testing robustness. Simpson's diversity index $(D)$ is commonly used to measure the degree of dominance of variants in the community ${ }^{32}$. It is a simple mathematical measure that characterizes diversity in a data set. The proportion of variants relative to the total number of variants $\left(p_{i}\right)$ is calculated and squared. The squared proportions for all the variants are summed, and the complement is taken:

$$
D=1-\sum_{i=1}^{R} p_{i}^{2}
$$

where $R$ corresponds to the total number of existing variants in the system (richness). $D$ ranges from 0 (one variant dominates the cultural system completely) to 1 (all variants are equally present).

\section{Results}

\section{Standard model: Initial random assignment of variants with replacement (wR)}

We first considered a model in which agents were initially assigned a cultural variant selected from a pool of variants with replacement. Simulation outcomes show that the co-evolutionary processes of value systems, cognitive biases and institutions implemented in the model tend to stabilize the diversity of the produced variants over time.

Figures 4 and 5 show the evolution of the cultural diversity of the set of produced variants at each round for a selection of representative parameter combinations of institutional influence $(\varepsilon)$, compliance bias $(\kappa)$, confirmation bias $(\gamma)$ and content bias $(\beta)$. Figures 6 and 7 show the evolution of the diversity of institutional values over time.

In general, $\varepsilon$ facilitates the emergence of shared cultural conventions when $\kappa>0$. This effect is amplified or minimised depending on how diverse institutions are at each time step. When $\varepsilon=1$, institutions have an immense capacity to convey their values effectively to the agents. In this scenario, when institutions are less diverse, convergence on shared cultural conventions is faster and diversity reduction stronger. This can be observed when we compare results of simulations using PR, where each agent was initialised with a randomised value system, against simulations using OTA, where agents where initialised with an homogeneous and hegemonic value system. As figures 4 and 5 show, diversity of produced variants is lower under OTA, and this is due to the reinforcing role of institutions, which push diversity up (PR) or down (OTA) according to how diverse their own value systems (the ones that they convey to the society) are. In general, content bias $(\beta)$ amplifies the effect of any parameter combination that allows the emergence of asymmetries in the dispersion of values among the set of existing cultural variants.

Interestingly, under $P R$ and $\varepsilon=1$, fully conformist populations $(\kappa=1)$ yield lower levels of convergence than populations with intermediate levels of $\kappa$. This result seems counterintuitive, but it is explained by the intrinsic properties of the institution: the emergent institution in this scenario is highly diverse and also has capacity to influence the agents' choices, resulting in a relatively high diversity of produced variants for all levels of content bias $\beta$. In other words, a compliance bias pushes diversity up when institutions are diverse, and pushes diversity down when institutions convey value systems with low diversity and strong dominance of one or few cultural variants. 
Intermediate values of $\varepsilon$ drastically reduce the formation of cultural conventions when compared to high levels of $\varepsilon$, and this effect increases with $\kappa$. Interestingly, a decrease in $\varepsilon$ increases diversity in both scenarios, PR and OTA, regardless of the diversity of institutional values promoted by the institution. Indeed, we can expect diverse institutions, like those that emerge in PR, to boost diversity. But, how is it possible that institutions in OTA, which convey extremely unambiguous and hegemonic value systems, also increase diversity. The answer lies in the strength with which these values, whether diverse or not, are transmitted to the population by the institutions. When $\varepsilon=0.5$ and $\kappa=1$, institutions are just partially successful conveying their values. This leads to a weaker transmission of institutional values over time (i.e. on average, the institutional value assigned to each existing cultural variant loses value at each time step). This combined with a population of agents willing to nevertheless keep adopting institutional values, ends up weakening agents' value systems too. The result is a society where values fade over time, leading to a limit in which the change in the frequency of an existing variant in the population is due to random sampling of variants. However, when $\varepsilon=0.5$ and agents do not fully conform, $\kappa<1$, alternative options for the emergence of cultural conventions arise. This is the case when $\kappa=0.5$ and $\gamma<1$ : In this scenario, cultural conventions can emerge from the mutual reinforcement of local interactions and institutional values. This is because as agents cease to fully conform with the social norms promoted by the institution, they begin to assign value to variants according to what is produced in their local interactions, which produces a stable convergence equilibria according to $\beta$. In a relatively wide range of these intermediate situations the model can be thought of as the most realistic example of most human communities, where neither are institutions fully influential to direct the agents' choices, nor are agents fully conformist or fully non-conformist.

When $\varepsilon=0$, institutions have no capacity to convey their values to the agents, so agents end up converging due to local interactions or not converging at all. For example, agents can attain moderately to high levels of convergence on cultural conventions without any institutional intervention when $\varepsilon$ is 0 and $\gamma$ is very high. In this scenario, agents converge due to their capacity to coordinate in their local interactions. On the other hand, a reduction in $\gamma$ implies that the agents' value systems become more volatile and less dependent on their individual experiences, resulting in a decrease in convergence.

An unusual simulated case is when agents are fully conformist, $\kappa=1$, and institutions have no influence at all. In this scenario, the system tends to high diversity of produced variants because variant selection ends up being similar to a random choice among variants with null value. A model that can be thought of as a society of believers drifting around and looking for something to believe in. On the other extreme, when $\kappa=0$ and $\gamma=1$, we have a society where agents do not conform at all but are fully biased towards their prior beliefs. In this case, convergence on shared conventions is dependent on the degree of initial similarity between the agents' value systems. 


\section{$\mathrm{H}_{\mathrm{n}}$ of produced variants: $w R$ and $P R$}

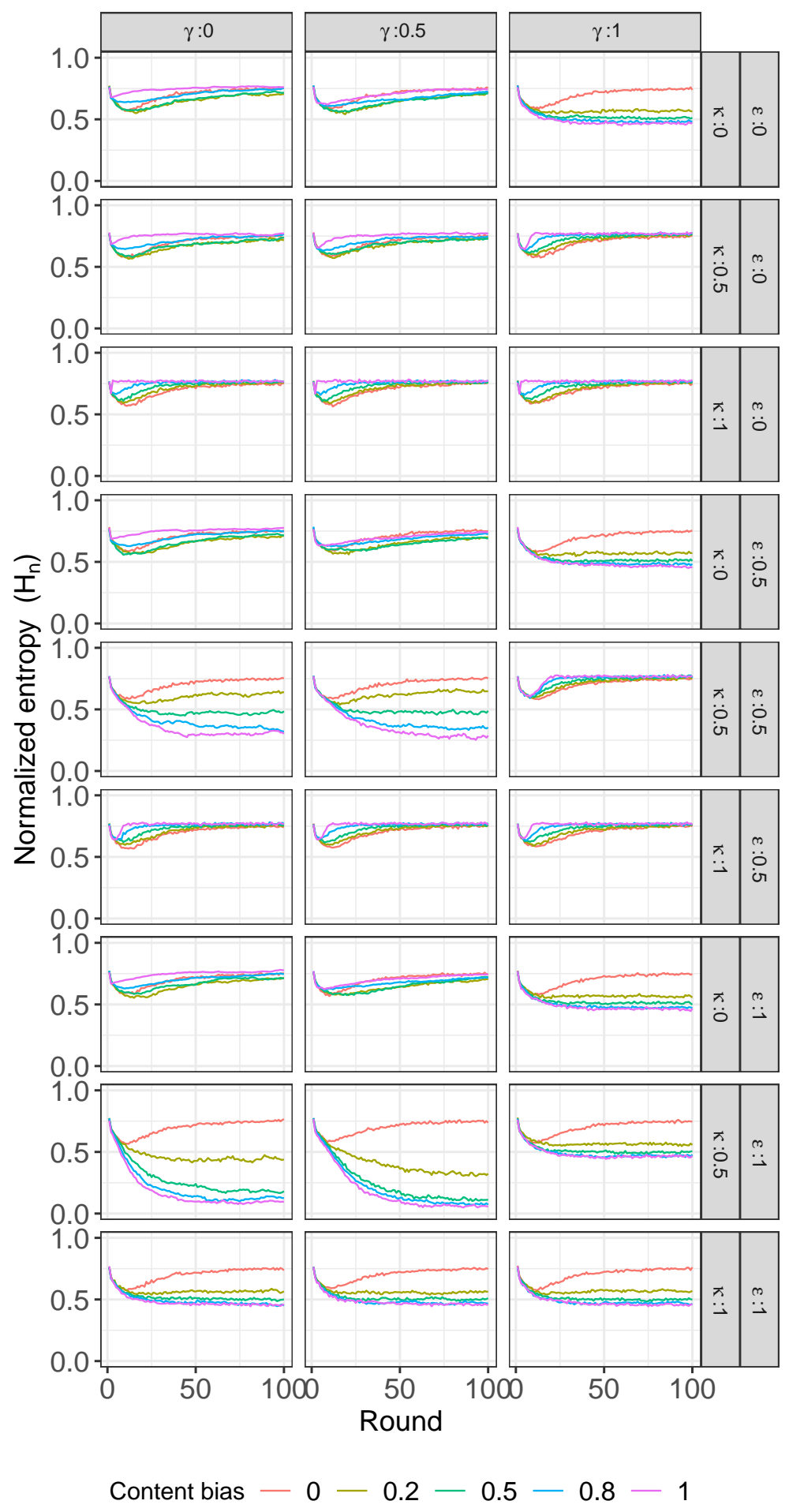

Figure 4. Cultural diversity (measured as Normalised Shannon Entropy $H_{n}$ ) of the set of produced variants over each level of institutional influence $(\varepsilon)$, compliance bias $(\kappa)$, confirmation bias $(\gamma)$ and content bias $(\beta)$. Simulations with initial random assignment of variants selected from $X$ with replacement (wR) and initial randomised value system (PR). 


\section{$\mathrm{H}_{\mathrm{n}}$ of produced variants: wR and OTA}

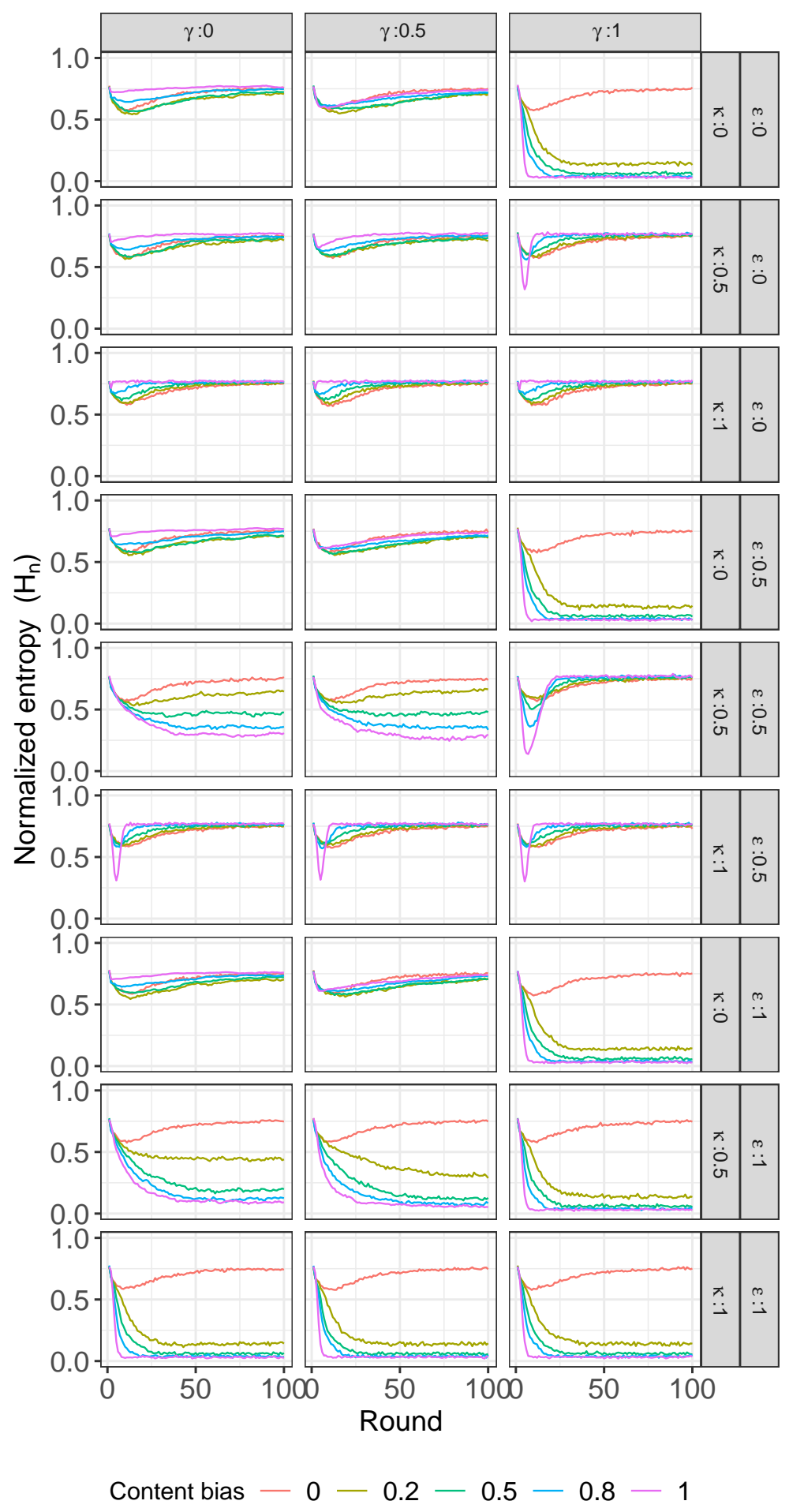

Figure 5. Cultural diversity (measured as Normalised Shannon Entropy $H_{n}$ ) of the set of produced variants over each level of institutional influence $(\varepsilon)$, compliance bias $(\kappa)$, confirmation bias $(\gamma)$ and content bias $(\beta)$. Simulations with initial random assignment of variants selected from $X$ with replacement (wR) and initial randomised value system (OTA). 


\section{$\mathrm{H}_{\mathrm{n}}$ of institutional values: $\mathrm{wR}$ and $\mathrm{PR}$}

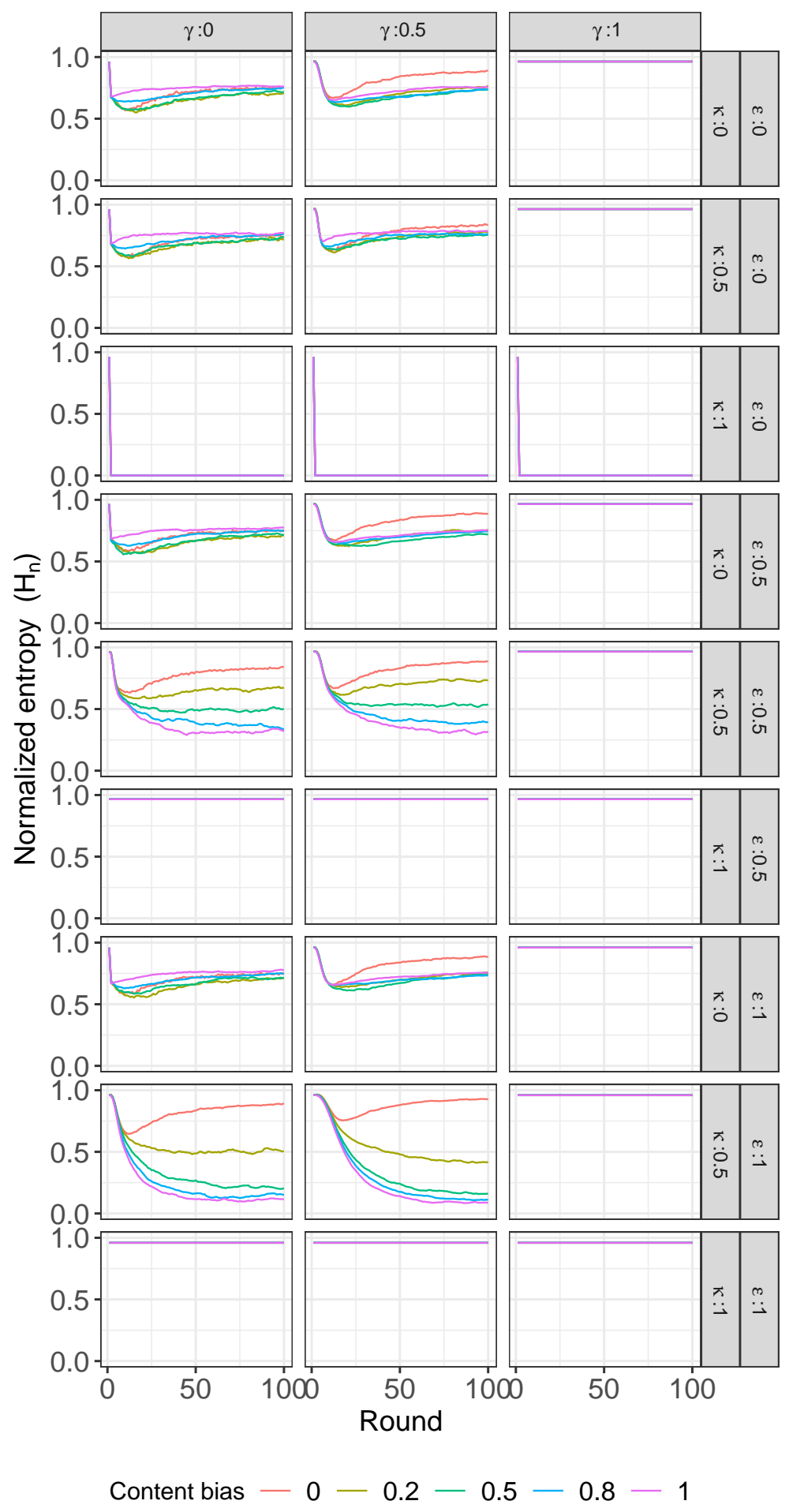

Figure 6. Diversity (measured as Normalised Shannon Entropy $H_{n}$ ) of institutional values over each level of institutional influence $(\varepsilon)$, compliance bias $(\kappa)$, confirmation bias $(\gamma)$ and content bias $(\beta)$. Simulations with initial random assignment of variants selected from $X$ with replacement (wR) and initial randomised value system (PR). 


\section{$\mathrm{H}_{\mathrm{n}}$ of institutional values: wR and OTA}

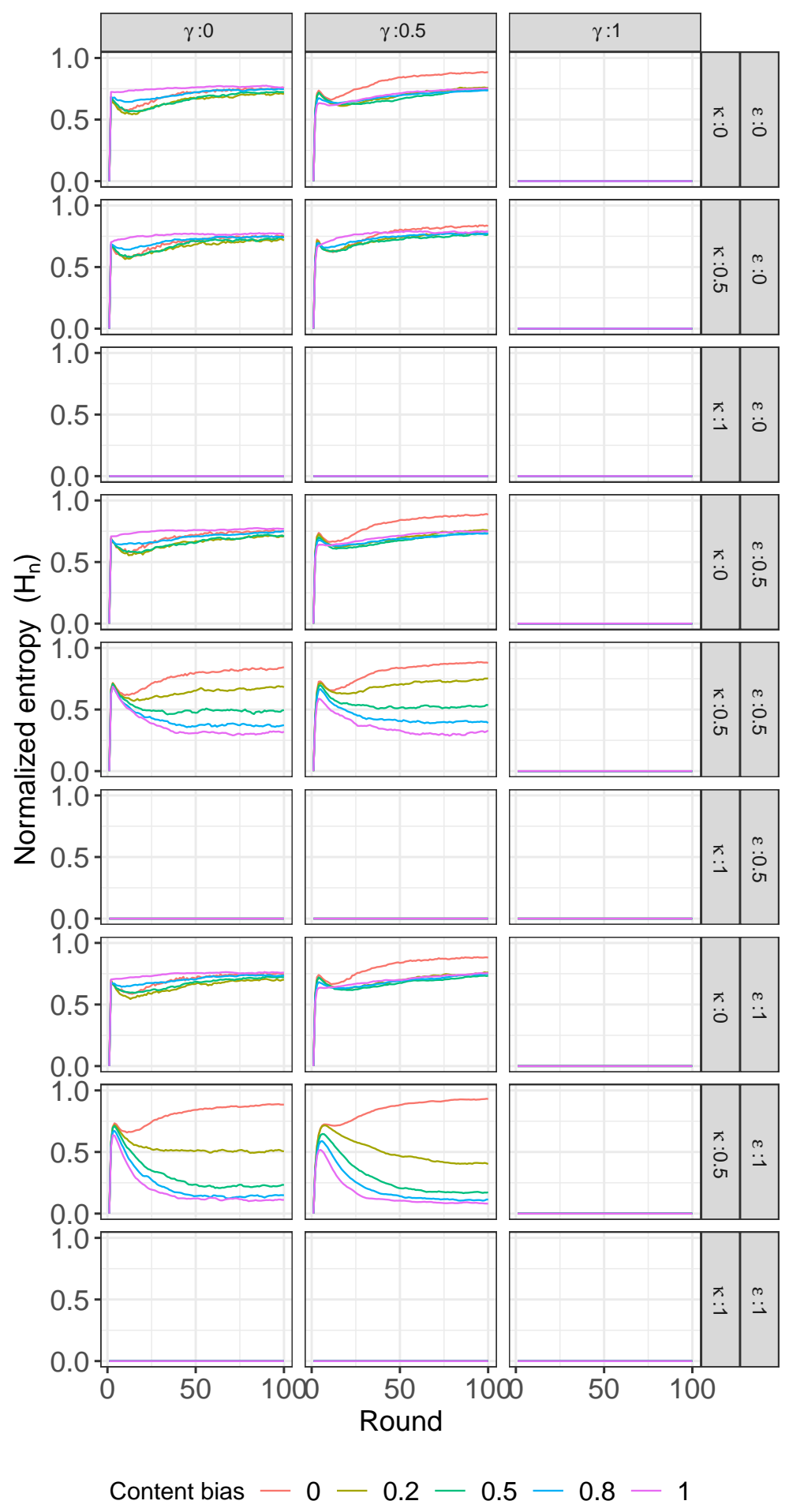

Figure 7. Diversity (measured as Normalised Shannon Entropy $H_{n}$ ) of institutional values over each level of institutional influence $(\varepsilon)$, compliance bias $(\kappa)$, confirmation bias $(\gamma)$ and content bias $(\beta)$. Simulations with initial random assignment of variants selected from $X$ with replacement (wR) and initial randomised value system (OTA). 


\section{Assumptions concerning initial variant assignment: Assignment without replacement}

Our model makes an important assumption about the initial state of diversity of cultural variants in the society, which depends on the method used to assign variants to agents at time 0 . In the simulations examined so far, each agent is initialised with a cultural variant randomly selected from a pool of $V$ distinct variants with replacement. This means that agents might share some variants in the initial state, resulting in a reduction of entropy. On average, this method yielded an initial diversity of around 0.75 (measured as Normalised Shannon Entropy, $H_{n}$ ). While we have shown that the co-evolution of value systems and institutions affect cultural diversity in different types of populations according to particular combinations of cognitive biases, we aim to asses the robustness of these findings and to what extent the model remains unchanged under different initial conditions of diversity. When agents are initialised with a cultural variant selected form a pool of variants without replacement, they start the simulation with a unique cultural variant, which means that diversity is the highest at time $0\left(H_{n}=1\right)$. However, in both conditions (with and without replacement), convergence on shared cultural conventions remains unchanged. Although diversity is initially lower when agents share variants from the beginning, mean diversity at equilibrium was the same in both conditions and remained unchanged in response to diversity perturbations, which implies that the system behaves consistently against these different assumptions; compare Figures 4 and 5 with Figures 8 and 9. The same consistency can be observed in regard to diversity of institutional values; compare Figures 6 and 7 with Figures 10 and 11). 


\section{$\mathrm{H}_{\mathrm{n}}$ of produced variants: woR and PR}

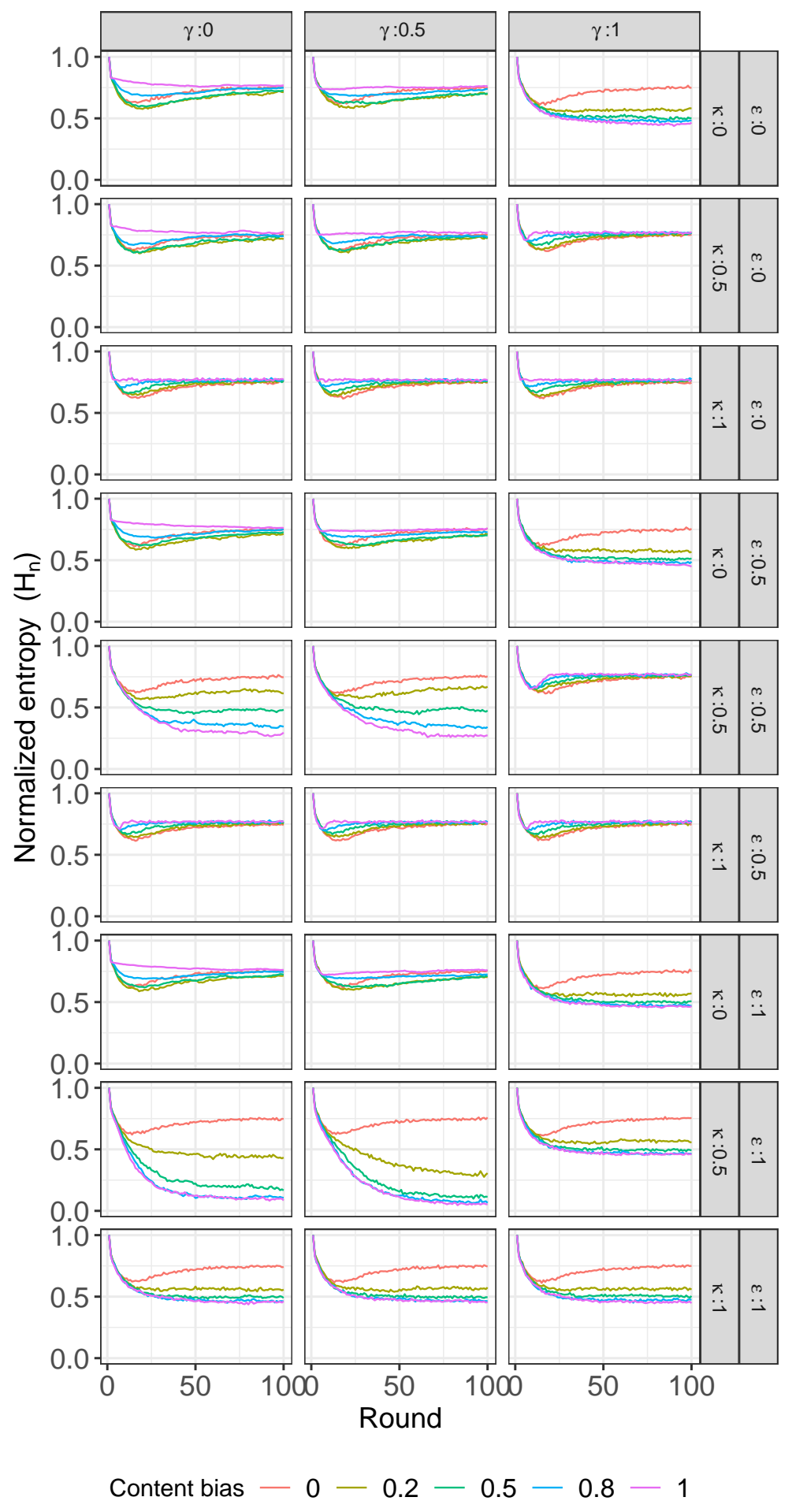

Figure 8. Cultural diversity (measured as Normalised Shannon Entropy $H_{n}$ ) of the set of produced variants over each level of institutional influence $(\varepsilon)$, compliance bias $(\kappa)$, confirmation bias $(\gamma)$ and content bias $(\beta)$. Simulations with initial random assignment of variants selected from $X$ without replacement (woR) and initial randomised value system (PR). 


\section{$\mathrm{H}_{\mathrm{n}}$ of produced variants: woR and OTA}

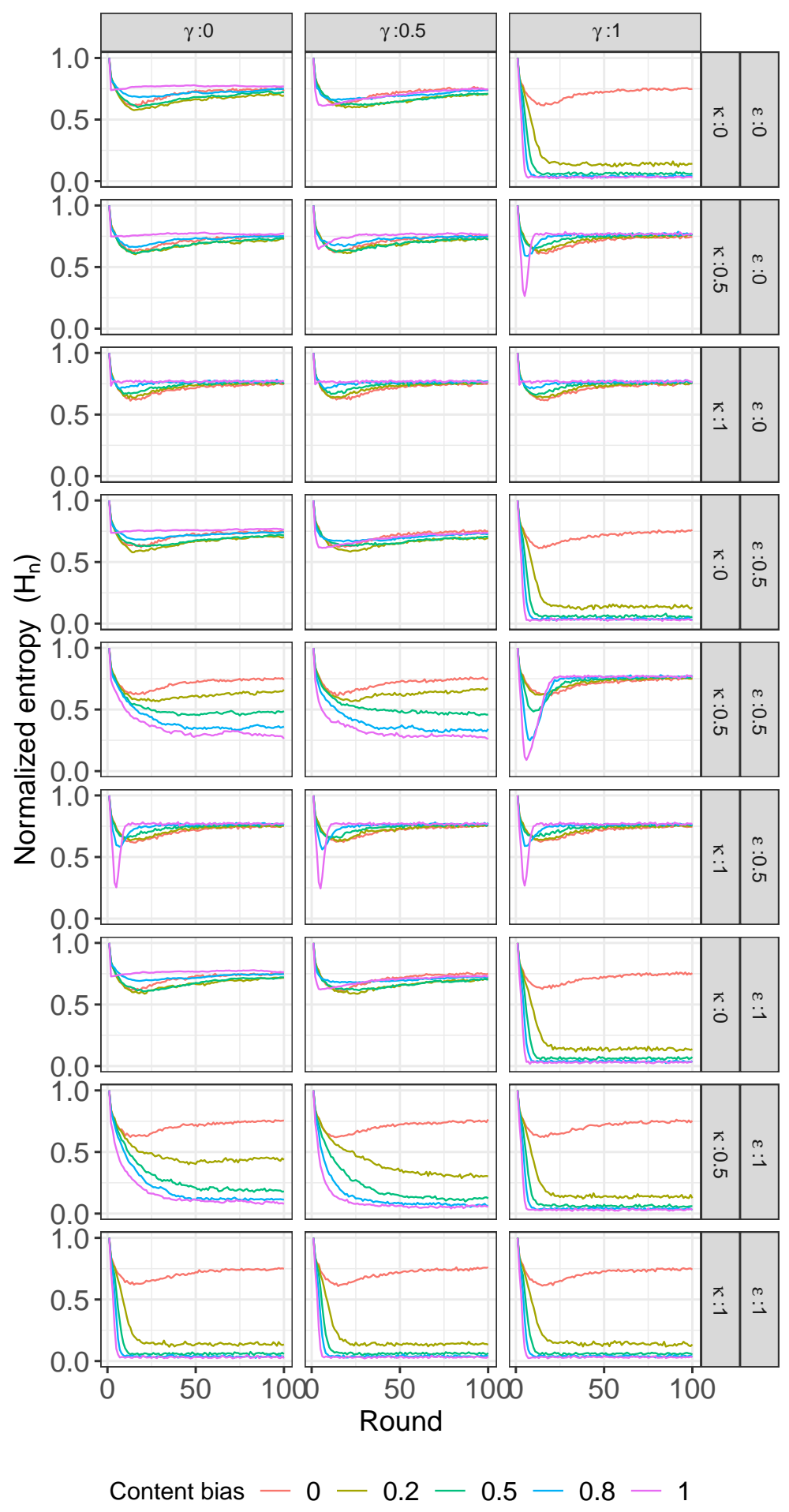

Figure 9. Cultural diversity (measured as Normalised Shannon Entropy $H_{n}$ ) of the set of produced variants over each level of institutional influence $(\varepsilon)$, compliance bias $(\kappa)$, confirmation bias $(\gamma)$ and content bias $(\beta)$. Simulations with initial random assignment of variants selected from $X$ without replacement (woR) and initial randomised value system (OTA). 


\section{$\mathrm{H}_{\mathrm{n}}$ of institutional values: woR and PR}

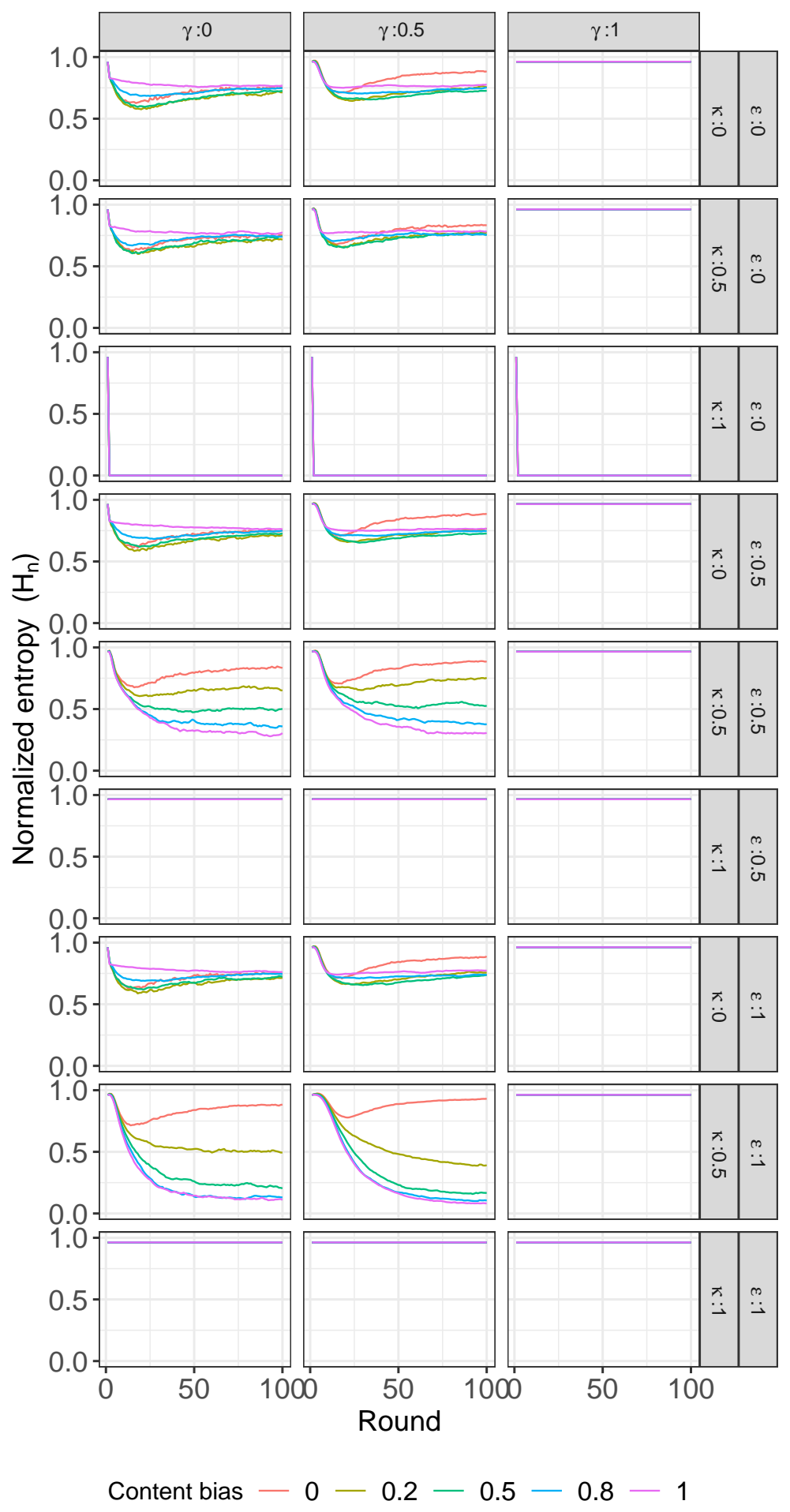

Figure 10. Diversity (measured as Normalised Shannon Entropy $H_{n}$ ) of institutional values over each level of institutional influence $(\varepsilon)$, compliance bias $(\kappa)$, confirmation bias $(\gamma)$ and content bias $(\beta)$. Simulations with initial random assignment of variants selected from $X$ without replacement (woR) and initial randomised value system (PR). 


\section{$H_{n}$ of institutional values: woR and OTA}

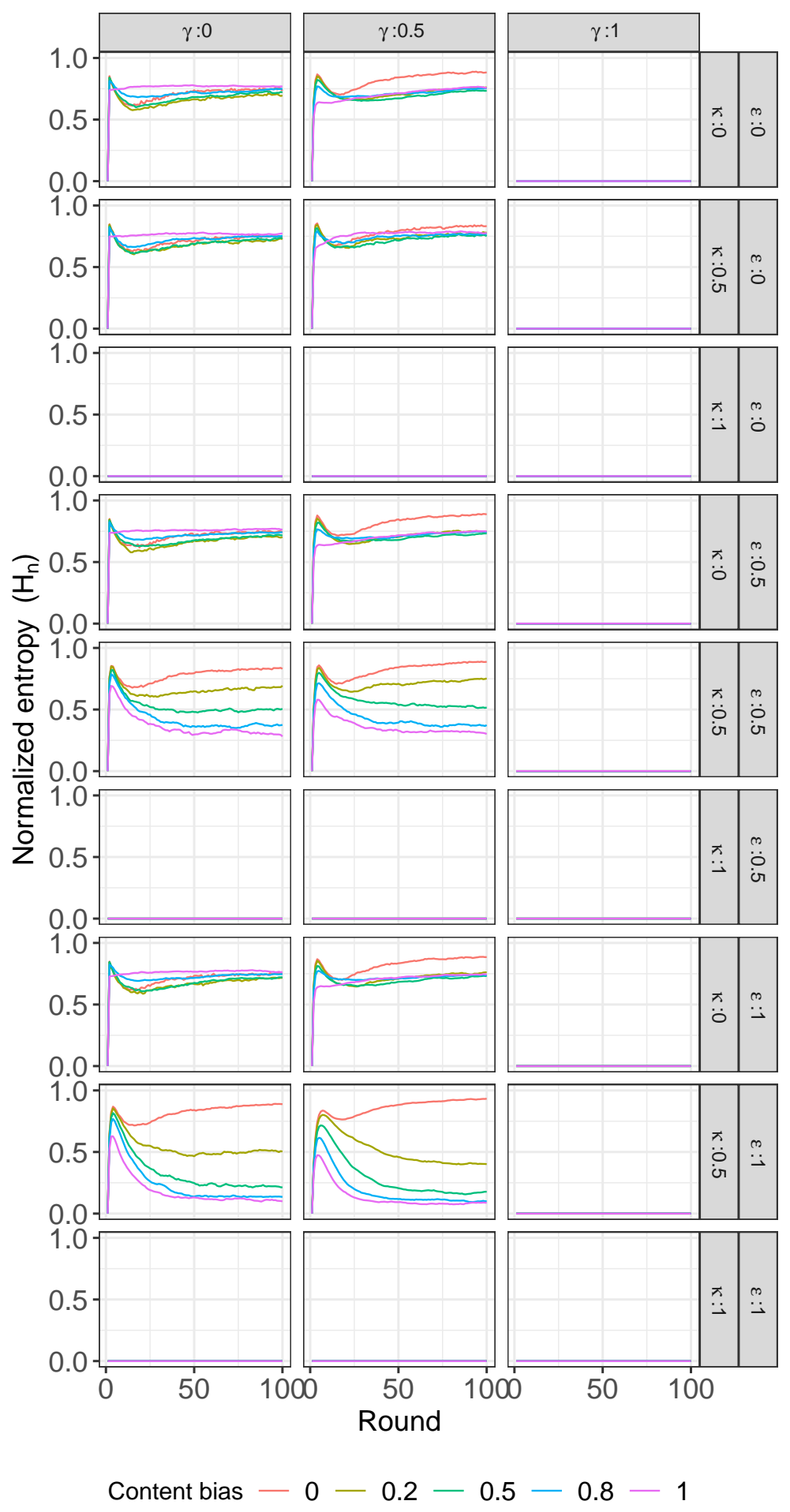

Figure 11. Diversity (measured as Normalised Shannon Entropy $H_{n}$ ) of institutional values over each level of institutional influence $(\varepsilon)$, compliance bias $(\kappa)$, confirmation bias $(\gamma)$ and content bias $(\beta)$. Simulations with initial random assignment of variants selected from $X$ without replacement (woR) and initial randomised value system (OTA). 


\section{Population size}

When population size increases, the emergence of shared cultural conventions is delayed, but the model yields qualitatively similar results for the population sizes examined. Figure 12 shows that the relative diversity of cultural variants remains higher in bigger populations until they eventually find their equilibrium. After 100 rounds, diversity outcomes are similar in both conditions. This is particularly true when content bias is greater than $0(\beta>0)$, because it allows agents to be sensitive towards variants value and, in turn, find similar equilibria under conditions of small and big population sizes. In contrast, for neutral content models $(\beta=0)$ differences between conditions are greater, with smaller populations reaching higher convergence. This is simply because the smaller the population, the higher the probability of sharing a variant by chance. Interestingly, in 100 agent micro-societies, when institutional influence is limited (intermediate values of $\varepsilon$ ), time to equilibrium is longer. This is due to the combined effect of interacting forces that simultaneously push diversity up and down, which results in a complex system that delays the formation of conventions even longer. In the present example, institutions are diverse and weak (which pushes diversity up) and agents individual biases are moderate (which slightly pushes diversity down due to alignment in the local interactions). In other words, in bigger populations, intermediate levels of institutional influence combined with intermediate levels of individual biases produce complex dynamics of antagonistic forces which translate into more time to reach consensus. This result implies that population size has an effect on model outcome. However, model results are still very similar qualitatively, which means that they hold for the range of populations sizes considered under the aforementioned assumptions.

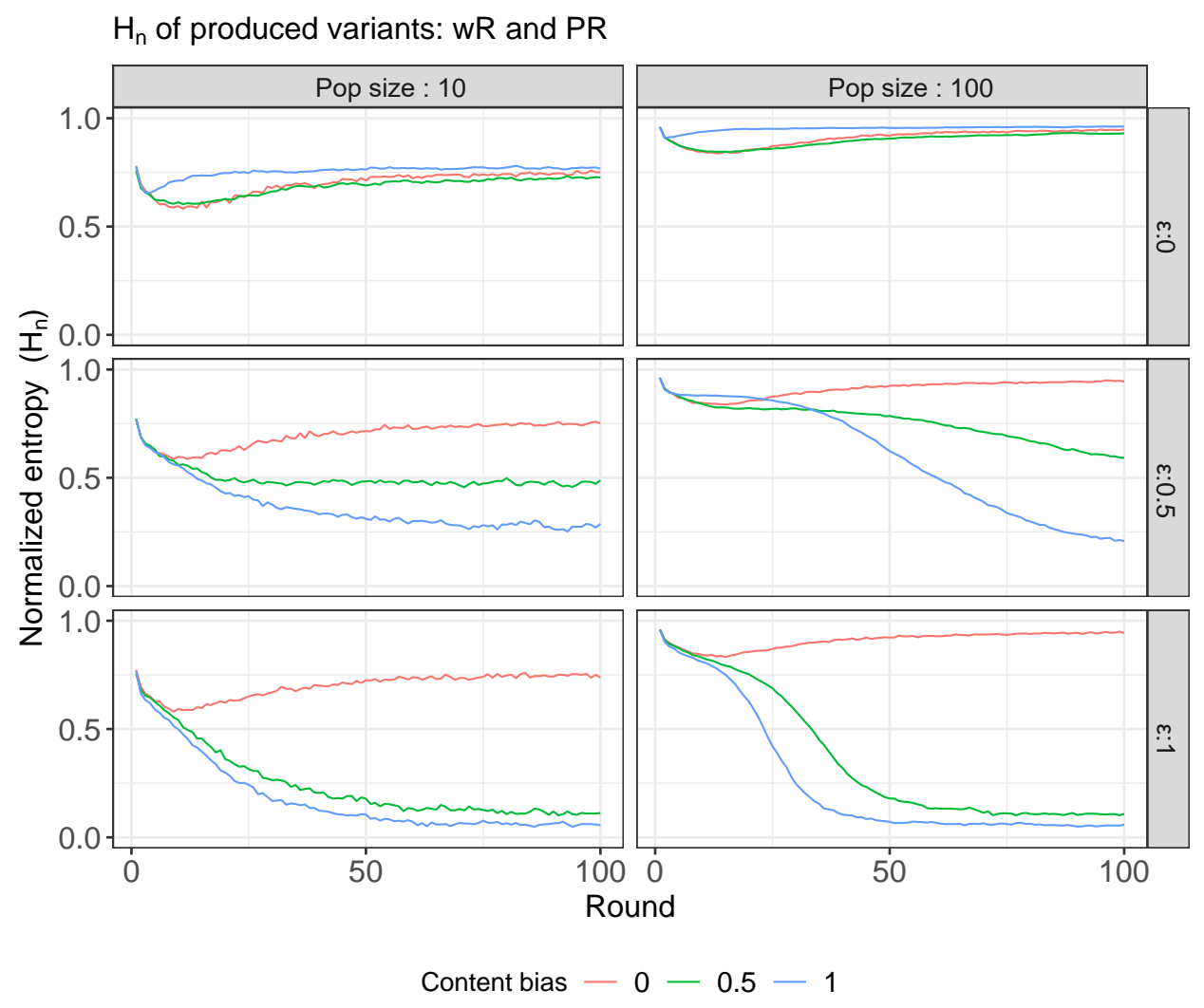

Figure 12. Cultural diversity (measured as Normalised Shannon Entropy, $H_{n}$ ) of the set of produced variants over time in 10 and 100 agents micro-societies. Results of three representative scenarios of institutional influence $(\varepsilon)$. Each scenario shows three different levels of content bias. Other cognitive biases were fixed at 0.5 . Simulations were initialised with a random assignment of variants selected from $X$ with replacement (wR) and a randomised value system (PR). 


\section{Alternative alpha diversity indexes}

A possible concern with the results of our study is that different diversity indexes might yield opposite trends. So far, we have used Shannon entropy $\left(H_{n}\right)$ as a measure of diversity. Richness (i.e. simply a count of variant types) and Simpson's diversity index, are also widely used in ecology studies. While the Shannon index stresses the richness component and the unpredictability of variant types, the Simpson index gives more weight to the evenness component and the degree of dominance of variant types. These differential emphases on different aspects of diversity might give rise to the possibility of non-conformance of diversity indexes, as has been demonstrated in the past $^{33,34}$.

In order to address this potential issue we also computed on Richness $(R)$ and Simpson's diversity index $(D)$ for a number of representative scenarios using the standard model (Figures 13 and 14). Results using these alternative alpha diversity indexes are fully consistent with our previous analyses. Model outcomes do not show opposite trends in response for the Shannon, Simpson and Richness indexes. Nevertheless, these additional analyses add relevant information about the evolution of the composition of the pool of cultural variants at each time step. Differences between highest and lowest $R(\simeq 0.5)$ are smaller than differences in $H_{n}(\simeq 0.68)$ (e.g. see $R$ and $H_{n}$ when $\varepsilon=1$, $\kappa=0.5$ and $\gamma=0.5$ ), which suggests intense competition among the surviving cultural variants over time, along with a contained extinction probability in most of the scenarios examined. Simpson's $D$ also confirms our previous analyses. Parameter combinations that facilitate the emergence of social conventions (measured as a decrease in $H_{n}$ ) coincide with those where there are few dominant variants at equilibrium (low $D$ ). Although with several important exceptions we have already explained, this happens mostly for moderate and high values of $\varepsilon, \kappa$ and $\beta$. 
Richness: wR and PR

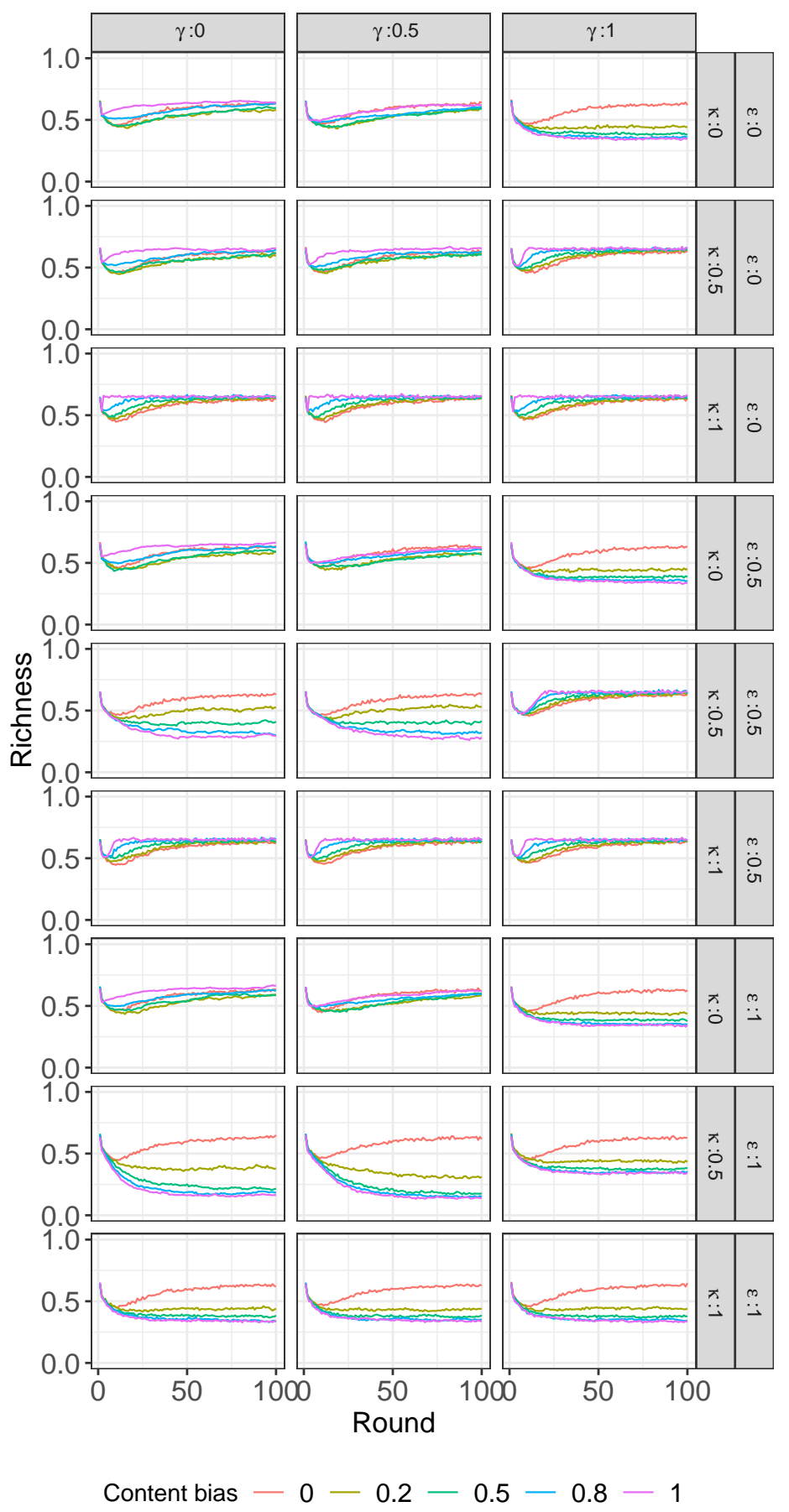

Figure 13. Richness (measured as the number of variants -operational taxonomic units- at each round) over each level of institutional influence $(\varepsilon)$, compliance bias $(\kappa)$, confirmation bias $(\gamma)$ and content bias $(\beta)$. Simulations with initial random assignment of variants selected from $X$ without replacement (wR) and initial randomised value system (PR). 


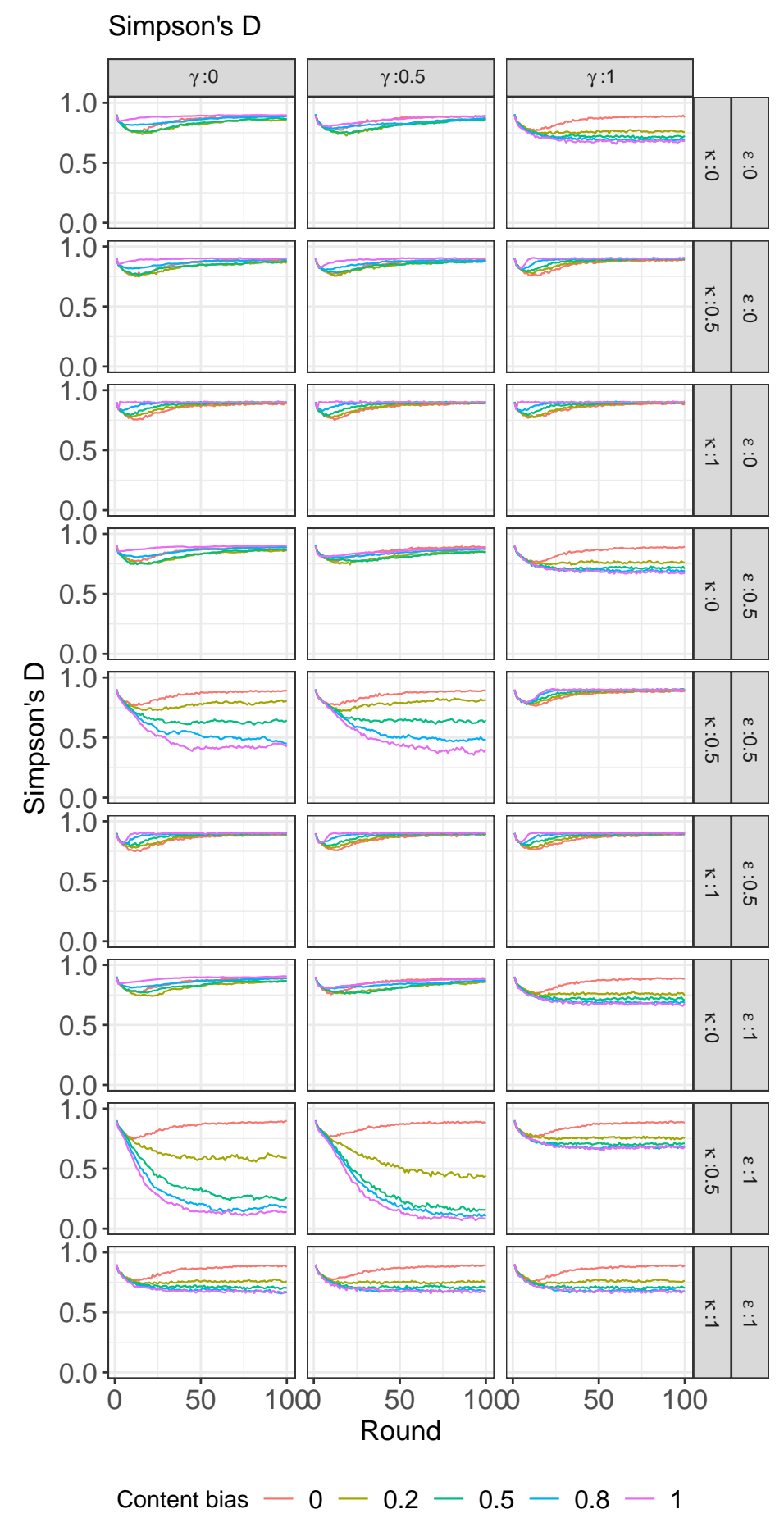

Figure 14. Diversity (measured as Simpson's diversity index $D$ ) of the set of produced variants over each level of institutional influence $(\varepsilon)$, compliance bias $(\kappa)$, confirmation bias $(\gamma)$ and content bias $(\beta)$. Simulations with initial random assignment of variants selected from $X$ without replacement (wR) and initial randomised value system (PR). 


\section{Discussion}

We have provided the first detailed analysis of the co-evolution of diversity of cultural variants, institutional values and agents' value systems for varying conditions of compliance, confirmation, content and frequency biases, and for different levels of institutional influence.

Institutional values and their capacity to convey those values to the population affects the agents' choices and in turn the diversity of cultural variants. In general, institutional influence facilitates the emergence of shared cultural conventions. These results generally confirm previous studies suggesting that institutions facilitate the emergence of social conventions ${ }^{6-9}$, but they also show that influential institutions can play an important role in the maintenance of cultural diversity. When institutions promote diversity and agents conform to the values promoted by institutions, the institutional capacity to transmit values efficiently becomes a prominent mechanism for the persistence of cultural diversity.

These results, however, are dependent on the institutional influence and the cognitive biases of the population. In order to achieve high levels of convergence, societies with influential institutions require agents to be at least moderately compliant, moderately content biased and moderately prone to consider new information to update their value systems. In the absence of institutional influence and therefore of institutions that are in place to guide convergence, strong conventions can also emerge. This is consistent with previous studies showing that social conventions can emerge without the intervention of institutional mechanisms ${ }^{5}$. However, in this scenario, high levels of convergence require a totally different combination of cognitive biases and value systems. Our model shows that, in the absence of institutional influence, societies require more hegemonic and homogeneous values at the population level, higher levels of confirmation bias and lower levels of compliance to form strong cultural conventions. In this scenario, the agents' value systems are similar and they are additionally reinforced by a confirmation bias in the local interactions. Agents, therefore, end up having strong and similar values. As a result of this, population convergence on shared conventions increases quickly as content bias increases.

Institutional diversity arises from the diversity of the agent's value systems. The more diverse the agents' values are the more diverse institutions are. This is unsurprising, because it responds to the way in which the model is constructed. What is noteworthy is that institutions with unambiguous and hegemonic value systems can also increase diversity. However, this only happens when institutions are partially successful at conveying their values: When transmission weakens institutional values and agents have a moderate-high compliance bias, the result is that population value systems also fade over time and in turn cultural diversity skyrockets. Nevertheless, societies with moderately influential institutions (either diverse or hegemonic) can stabilize the diversity of the produced variants at intermediate levels when agents are moderately compliant, especially when agents have a moderate-high tendency to consider new information to update their prior value systems. These results show that institutions, the agents' values and biases interact affecting the dynamics of social conventions: Different institutions can yield different or similar levels of diversity depending on how they interact with the agents' cognition.

Formal models show that conformity with institutional norms or beliefs can safeguard cultural diversity from erosion $^{15}$. In particular, strong conformity seems to affect human culture by facilitating stable in-group uniformity, which then stabilises between-group cultural diversity over time ${ }^{35,36}$. In our model, compliance with institutional value systems works in a similar way in some scenarios: For example, when $\varepsilon=1$ and the population behaves as an in-group (homogeneous and hegemonic value systems (OTA), an increase in diversity tends to increase convergence. In this context, when the population forms an out-group (PR), cultural diversity tends to increase when we move from intermediate levels to high levels of $\kappa$. Our model, therefore, suggests that compliance can be considered a prominent mechanism underlying the stabilization of cultural diversity.

However, our results also show that cultural diversity can be maintained without an individual compliance bias. This is particularly true when $\gamma<1$, that is, when agents are more prone to use new information to update their value systems. Previous studies have suggested the possibility that the maintenance of the stability of cultural diversity could occur without a conformity bias ${ }^{20}$. An interesting direction for future research would be to implement a conformity bias (disproportionate tendency to copy the majority) and test the results against the compliance bias implemented here. This would allow us to check whether a lack of confirmation bias, in combination with other 
evolutionary pressures, is a candidate for the preservation of cultural diversity in scenarios without an individual-level conformity bias.

Interestingly, in some scenarios (intermediate values of $\varepsilon$ and $\kappa$ ), our study is consistent with recent studies showing that confirmation bias is determinant for the formation of communities of common interests, which in turn rises cultural variation ${ }^{24}$. In contrast to these results, in particular in PR scenarios, our simulations show that a strong confirmation bias without a compliance bias tends to increase convergence. It is still unclear, however, whether a confirmation bias alone can drive convergence on shared conventions without endowing individuals with other cognitive biases related to the intrinsic value of the cultural traits-in our case, a general individual-level content bias.

To ensure that our results were not dependent on the range of initial values of cultural diversity, we ran simulations using an initial assignment of variants without replacement. For all conditions the results were qualitatively identical to the runs with initial assignment of variants with replacement. We also ran simulations using a populations of 10 and 100 agents. Simulations results were again very similar between conditions. Additionally, we quantified diversity outputs using a number of alternative well-established alpha diversity indexes and we verified that all the different metrics yielded similar results. All these robustness tests mean that our model holds for a wide range of critical assumptions.

\section{Conclusions}

This study has introduced institutions, value systems and a set of cognitive biases into the dynamics of cultural evolution. This co-evolutionary model formalises the idea that cultural diversity changes with the processes of cognitive and institutional interaction. The interaction between cognitive biases, value systems and institutions reveal a complex relationship between individual-level compliance, confirmation bias and value systems and institutionallevel values and influence. In general, institutional influence facilitates the formation of cultural conventions. However, institutional influence can increase cultural diversity when agents are compliant and institutions promote diverse values.

In the absence of institutions that guide convergence strong conventions can also emerge. In our simulations, this occurs when populations of non-compliant agents have similar value systems and are endowed with strong individual-level confirmation bias. In more realistic scenarios, where institutional influence is intermediate, a moderate compliance bias can be a potent mechanism underlying the stabilization of cultural diversity at intermediate levels.

The results presented in this study might be of interest to researchers investigating the impact of representative institutions on the evolution of linguistic, cultural, economic or social behavior ${ }^{4,5,13,27,37}$. In particular, an interesting research question for future work will be to explore in a formal model the relationships between conformity and compliance biases, how they interact and how they drive the emergence of cultural conventions in in-groups and out-groups. Future work using our co-evolutionary model will also be focused on mathematical modelling for a wider range of institutions.

\section{References}

1. Laland, K. N., Odling-Smee, J. \& Feldman, M. W. Niche construction, biological evolution, and cultural change. Behav. Brain Sci. 23, 131-146, DOI: https://doi.org/10.1017/S0140525X00002417 (2000).

2. Fuentes, A., Wyczalkowski, M. A. \& MacKinnon, K. C. Niche construction through cooperation: a nonlinear dynamics contribution to modeling facets of the evolutionary history in the genus homo. Curr. Anthropol. 51, 435-444 (2010).

3. Kobayashi, Y., Wakano, J. Y. \& Ohtsuki, H. Evolution of cumulative culture for niche construction. J. theoretical biology 472, 67-76 (2019).

4. Bowles, S., Choi, J.-K. \& Hopfensitz, A. The co-evolution of individual behaviors and social institutions. $J$. theoretical biology 223, 135-147 (2003). 
5. Becker, J., Brackbill, D. \& Centola, D. Network dynamics of social influence in the wisdom of crowds. Proc. national academy sciences 114, E5070-E5076 (2017).

6. Young, H. P. Individual strategy and social structure: An evolutionary theory of institutions (Princeton University Press, 2001).

7. Voigt, S. \& Kiwit, D. The role and evolution of beliefs, habits, moral norms, and institutions. In Merits and limits of markets, 83-108 (Springer, 1998).

8. David, P. A. Why are institutions the 'carriers of history'?: Path dependence and the evolution of conventions, organizations and institutions. Struct. change economic dynamics 5, 205-220 (1994).

9. Kearns, M., Judd, S., Tan, J. \& Wortman, J. Behavioral experiments on biased voting in networks. Proc. Natl. Acad. Sci. 106, 1347-1352 (2009).

10. Lewontin, R. Selection in and of populations. ideas in modern biology. In Proc. XVI Int. Cong. Zool., vol. 6, 299-311 (1965).

11. Kendal, J., Tehrani, J. J. \& Odling-Smee, J. Human niche construction in interdisciplinary focus (2011).

12. Levinson, S. C. \& Levinson, S. C. Space in language and cognition: Explorations in cognitive diversity, vol. 5 (Cambridge University Press, 2003).

13. Henrich, J. et al. "economic man" in cross-cultural perspective: Behavioral experiments in 15 small-scale societies. Behav. brain sciences 28, 795-815 (2005).

14. Henrich, J., Heine, S. J. \& Norenzayan, A. The weirdest people in the world? Behav. brain sciences 33, 61-83 (2010).

15. Boyd, R. \& Richerson, P. Culture and the evolutionary process. chicago: Univ. Press. Chic. (1985).

16. Feldman, M. W. \& Cavalli-Sforza, L. L. Cultural and biological evolutionary processes, selection for a trait under complex transmission. Theor. Popul. Biol. 9, 238-259, DOI: https://doi.org/10.1016/0040-5809(76)90047-2 (1976).

17. Walker, B., Segovia Martín, J., Tamariz, M. \& Fay, N. Maintenance of prior behaviour can enhance cultural selection. Sci. reports 11, 1-9 (2021).

18. Henrich, J. \& McElreath, R. The evolution of cultural evolution. Evol. Anthropol. Issues, News, Rev. Issues, News, Rev. 12, 123-135 (2003).

19. Cialdini, R. B. \& Goldstein, N. J. Social influence: Compliance and conformity. Annu. Rev. Psychol. 55, 591-621 (2004).

20. Acerbi, A., Van Leeuwen, E. J., Haun, D. B. \& Tennie, C. Conformity cannot be identified based on populationlevel signatures. Sci. reports 6, 36068 (2016).

21. Morgan, T. J. H. \& Laland, K. N. The biological bases of conformity. Front. neuroscience 6, 87 (2012).

22. Asch, S. E. Studies of independence and conformity: I. a minority of one against a unanimous majority. Psychol. monographs: Gen. applied 70, 1 (1956).

23. Morgan, T. J., Acerbi, A. \& Van Leeuwen, E. J. Copy-the-majority of instances or individuals? two approaches to the majority and their consequences for conformist decision-making. PloS one 14 (2019).

24. Del Vicario, M., Scala, A., Caldarelli, G., Stanley, H. E. \& Quattrociocchi, W. Modeling confirmation bias and polarization. Sci. reports 7, 40391 (2017).

25. Quattrociocchi, W., Scala, A. \& Sunstein, C. R. Echo chambers on facebook. Available at SSRN 2795110 (2016).

26. Nickerson, R. S. Confirmation bias: A ubiquitous phenomenon in many guises. Rev. general psychology $\mathbf{2}$, 175-220 (1998). 
27. Centola, D. \& Baronchelli, A. The spontaneous emergence of conventions: An experimental study of cultural evolution. Proc. Natl. Acad. Sci. 112, 1989-1994 (2015).

28. Segovia-Martin, J. \& Tamariz, M. Synchronising institutions and value systems: A model of opinion dynamics mediated by proportional representation. PLOS ONE 16, 1-31, DOI: 10.1371/journal.pone.0257525 (2021).

29. Tamariz, M., Ellison, T. M., Barr, D. J. \& Fay, N. Cultural selection drives the evolution of human communication systems. Proc. Royal Soc. B: Biol. Sci. 281, 20140488 (2014).

30. Fay, N., Garrod, S., Roberts, L. \& Swoboda, N. The interactive evolution of human communication systems. Cogn. science 34, 351-386 (2010).

31. Segovia-Martín, J., Walker, B., Fay, N. \& Tamariz, M. Network connectivity dynamics, cognitive biases, and the evolution of cultural diversity in round-robin interactive micro-societies. Cogn. science 44, e12852 (2020).

32. Simpson, E. H. Measurement of diversity. Nature 163, 688 (1949).

33. Nagendra, H. Opposite trends in response for the shannon and simpson indices of landscape diversity. Appl. geography 22, 175-186 (2002).

34. Hurlbert, S. H. The nonconcept of species diversity: a critique and alternative parameters. Ecology 52, 577-586 (1971).

35. Henrich, J. \& Boyd, R. The evolution of conformist transmission and the emergence of between-group differences. Evol. human behavior 19, 215-241 (1998).

36. Henrich, N. \& Henrich, J. P. Why humans cooperate: A cultural and evolutionary explanation (Oxford University Press, 2007).

37. Boyd, R. \& Richerson, P. J. Gene-culture coevolution and the evolution of social institutions. Philpapers.org (2008). 